\title{
Novas espécies de aves para o Estado de Santa Catarina, sul do Brasil
}

\author{
Adrian Eisen Rupp ${ }^{1 *}$ \\ Daniela Fink ${ }^{2}$ \\ Gregory Thom e Silva ${ }^{1}$ \\ Morgana Zermiani \\ Rudi Ricardo Laps ${ }^{1,2,3}$ \\ Carlos Eduardo Zimmermann ${ }^{1}$
${ }^{1}$ Universidade Regional de Blumenau, Instituto de Pesquisas Ambientais, Laboratório de Ecologia e Ornitologia Rua Antônio da Veiga, 140, CEP 89010-971, Blumenau - SC
${ }^{2}$ Associação Catarinense de Preservação da Natureza ${ }^{3}$ Universidade Regional de Blumenau, Departamento de Ciências Naturais
*Autor para correspondência
eisenrupp@yahoo.com.br

Submetido em 19/02/2008

Aceito para publicação em 28/04/2008

\section{Resumo}

O conhecimento ornitológico em Santa Catarina aumentou consideravelmente nas últimas duas décadas, e com freqüência são realizadas adições à avifauna catarinense ou redescobertas de espécies com registros históricos. Desta forma, apresentamos os primeiros registros e localidades de ocorrência de Phimosus infuscatus, Accipiter poliogaster, Porzana flaviventer, Picumnus cirratus e Polioptila lactea em Santa Catarina.

Unitermos: Phimosus infuscatus, Accipiter poliogaster, Porzana flaviventer, Picumnus cirratus, Polioptila lactea, Santa Catarina

\section{Abstract}

Birds new to Santa Catarina State, southern Brazil. Ornithological knowledge in Santa Catarina has improved considerably in recent decades, and new records and rediscoveries of bird species known only by historical data have been made. As a result of field research, we present herein the first records and locations of the Bare-faced Ibis Phimosus infuscatus, Gray-bellied Hawk Accipiter poliogaster, Yellow-breasted Crake Porzana flaviventer, Creamy-bellied Gnatcatcher Polioptila lactea and White-barred Piculet Picumnus cirratus in Santa Catarina State.

Key words: Phimosus infuscatus, Accipiter poliogaster, Porzana flaviventer, Picumnus cirratus, Polioptila lactea, Santa Catarina State 
Santa Catarina tem recebido um incremento considerável no conhecimento ornitológico, principalmente devido à formação de novos ornitólogos e conseqüente aumento no número de pesquisas de campo. Desde Rosário (1996), novas informações sobre a riqueza de espécies, distribuição e status de conservação das aves do Estado foram adquiridas (e.g. Naka et al., 2000 e 2001; Amorim e Piacentini, 2006 e 2007; Ghizoni-Jr. e Silva, 2006; Piacentini et al., 2006). Muitos dos registros adicionais à avifauna catarinense são de espécies conhecidas em regiões adjacentes ao Estado, como na Argentina (Misiones), Paraná e Rio Grande do Sul (e.g. Accordi et al., 2002; Ghizoni-Jr., 2004; Azevedo e Ghizoni-Jr., 2005; Amorim e Piacentini, 2007; Rupp et al., 2007). Como resultado de trabalhos de campo em Santa Catarina, apresentamos cinco espécies inéditas ou sem localidades conhecidas, cuja presenca ja era esperada no estado.

Phimosus infuscatus (Lichtenstein, 1823) (tapicurude-cara-pelada): Espécie de ampla distribuição na América do Sul, meridionalmente até o Uruguai e Argentina (Sick, 1997). No Rio Grande do Sul, é encontrada na Campanha gaúcha e banhados do litoral em todos os meses do ano (Belton, 1994). Em Santa Catarina encontramos esta espécie ao longo do litoral e baixo vale do Itajaí, em áreas onde a vegetação natural foi substituída por pastagens e arrozeiras, e também em lagoas naturais e em manguezais, sendo observada nas margens da rodovia BR-101 em várias ocasiões (Tabela 1), inclusive em áreas de trânsito intenso em Florianópolis. Estes são os primeiros registros publicados de $P$. infuscatus em Santa Catarina, e muitos destes em áreas onde foram realizados vários estudos (Bege e Marterer, 1991; Rosário 1996; Naka et al., 2002; Piacentini e Campbell-Thompson, 2006; Efe et al., 2007). Isto sugere uma possível colonização recente no Estado, ou um aumento populacional decorrente da transformação da paisagem, onde a Floresta Ombrófila Densa das Terras Baixas foi ocupada e substituída por arrozeiras e pastagens, aumentando a área de forrageio da espécie.

Accipiter poliogaster (Temminck, 1824) (tauatópintado): Reichholf (1974) menciona três indivíduos registrados em Santa Catarina em 1970, entretanto não fornece as localidades. Rosário (1996) também não apresenta registros enquanto que Sick et al. (1981) relatam apenas registros bibliográficos para a inclusão da espécie em SC. Esta espécie é conhecida por registros pontuais ao longo de sua distribuição, sendo que meridionalmente é encontrada principalmente na Floresta Ombrófila Mista e na Floresta Estacional Semidecidual (Belton, 1994; Straube et al., 2004). Registramos a espécie em duas ocasiões na Reserva Biológica Estadual do Sassafrás, município de Doutor Pedrinho, no dia 12 de dezembro de 2006 (2642'45"S e $49^{\circ} 39^{\prime} 58^{\prime \prime}$ W, 838 m.s.n.m.) e no dia 26 de agosto de 2007 (26²'42”'S e 4940'32”W, 957 m.s.n.m.), em uma área de transição entre a Floresta Ombrófila Densa e Mista. Os indivíduos foram identificados pelo porte, sendo maiores que Accipiter bicolor (também registrada na Reserva), pela coloração da região dorsal e coroa de tonalidade cinza escuro, presença de três barras na cauda, e pela ausência de calções ferrugíneos. Estas características tornam possível a identificação de Accipiter poliogaster em campo e estão de acordo com as descrições da plumagem desta espécie (Sick, 1997; De La Peña e Rumboll, 1998; Ferguson-Lees e Christie, 2001; Straube et al., 2004). Esta é a primeira localidade com registros da espécie em Santa Catarina.

Porzana flaviventer (Boddaert, 1783) (sanãamarela): Este Rallidae é encontrado desde a América Central e Caribe até a Argentina na América do Sul (Sick, 1997), onde ocorre de modo pontual considerando os registros ocasionais no Paraná (Bornschein et al., 1997) e Rio Grande do Sul (Belton, 1994). No dia 21 de novembro de 2006 um indivíduo de P. flaviventer foi encontrado morto em uma arrozeira próxima à área urbana do município de Rodeio. O espécime está depositado na coleção da Universidade Regional de Blumenau (FURB 10936).

Picumnus cirratus (Temminck, 1825) (pica-pauanão-barrado): Rosário (1996) apresenta registros desta espécie em todo o Estado. No entanto, esta segue a taxonomia adotada por Sick (1993) que considera o táxon P. temminckii subespécie de P. cirratus. O Comitê Brasileiro de Registros Ornitológicos considera estes dois taxa como espécies independentes (CBRO, 2001), e sugere que a distribuição geográfica destas espécies deve 
ser redefinida (CBRO, 2002). Nenhum dos registros de $P$. cirratus apresentados por diversos autores em Santa Catarina define seu status subespecífico como sendo diferente de P. temminckii, assim como todos os espécimes depositados na coleção Zoológica da FURB se referem a P. temminckii (FURB 1502, 1503, 1504, 1505, 1953, 10738, 10963), impossibilitando afirmativas quanto à ocorrência de $P$. cirratus no estado. Os registros no Rio Grande do Sul, apresentados por Albuquerque (1977, apud Belton, 1994), foram considerados hipotéticos pelo próprio autor (Bencke, 2001). No Paraná sua presença é confirmada por duas peles coletadas no extinto Parque Nacional de Sete Quedas e depositadas no Museu de História Natural Capão da Imbuia, e por registros no
Parque Estadual do Cerrado em Jaguariaíva (Straube et al., 2005). No dia 03 de novembro de 2006 encontramos um indivíduo macho de Picumnus cirratus na zona de amortecimento da REBIO Estadual do Sassafrás (2642'33"S e 4940'58'W, 954 m s.n.m.). A ave distava cerca de três metros dos observadores, favorecendo a observação de características diagnósticas, como a região dorsal de cor predominante cinza e ausência de colar nucal ferrugíneo. Pelo contrário, Picumnus temminckii apresenta o corpo cinza-amarronzado e colar nucal ferrugíneo (Belton, 1994; Sick, 1997; Develey e Endrigo, 2004). É importante mencionar que na região ocorrem Picumnus nebulosus (FURB 13202) e Picumnus temminckii.

TABELA 1: Municípios, locais, ambientes (FOD: Floresta Ombrófila Densa, MGZ: Manguezal, RES: Restinga, ARR: Arrozeira, PAS: Pastagem), coordenadas geográficas e data dos registros de Phimosus infuscatus em Santa Catarina, Brasil.

\begin{tabular}{|c|c|c|c|}
\hline Município e Local & Ambiente & Coordenadas & Data \\
\hline \multicolumn{4}{|l|}{ Blumenau } \\
\hline APA das Ilhas Fluviais do Rio Itajaí Açu & FOD & $26^{\circ} 53^{\prime} 35^{\prime}$ 'S e $49^{\circ} 08^{\prime} 24^{\prime \prime} \mathrm{W}$ & $\begin{array}{l}08 / 06 / 2006 \\
08 / 07 / 2006\end{array}$ \\
\hline \multicolumn{4}{|l|}{ Gaspar } \\
\hline SC-411 & ARR & $26^{\circ} 56^{\prime} \mathrm{S}$ e $48^{\circ} 56^{\prime} \mathrm{W}$ & $15 / 02 / 2006$ \\
\hline \multirow[t]{4}{*}{ Belchior Baixo } & ARR & $26^{\circ} 55^{\prime} 53^{\prime \prime}$ S e $48^{\circ} 57^{\prime} 32^{\prime \prime} \mathrm{W}$ & $14 / 07 / 2006$ \\
\hline & & & $17 / 08 / 2006$ \\
\hline & & & $01 / 09 / 2006$ \\
\hline & & & $20 / 09 / 2006$ \\
\hline BR-470 & ARR & & $26 / 12 / 2007$ \\
\hline \multicolumn{4}{|l|}{ Porto Belo } \\
\hline BR-101 & PAS & $27^{\circ} 09^{\prime} \mathrm{S}$ e $48^{\circ} 36^{\prime} \mathrm{W}$ & $29 / 07 / 2007$ \\
\hline Rio Perequê & MGZ & $27^{\circ} 08^{\prime} 46^{\prime \prime}$ S e $48^{\circ} 35^{\prime} 22^{\prime \prime} \mathrm{W}$ & $27 / 01 / 2007$ \\
\hline \multicolumn{4}{|l|}{ Florianópolis } \\
\hline Parque Est. Serra do Tabuleiro (Baixada do Maciambu) & RES & $27^{\circ} 50^{\prime} 31^{\prime \prime S}$ e $48^{\circ} 37^{\prime} 32^{\prime \prime} \mathrm{W}$ & $04 / 10 / 2007$ \\
\hline \multirow[t]{2}{*}{ Aterro da Costeira na enseada do Saco dos Limões } & - & $27^{\circ} 36^{\prime} 46^{\prime \prime}$ S e $48^{\circ} 31^{\prime} 50^{\prime \prime} \mathrm{W}$ & $29 / 07 / 2007$ \\
\hline & - & & $10 / 11 / 2007$ \\
\hline Estreito - BR-282 & PAS & $27^{\circ} 35^{\prime} 37^{\prime}$ 'S e $48^{\circ} 36^{\prime} 06^{\prime \prime W}$ & $06 / 11 / 2007$ \\
\hline BR-101 (Palhoça até Balneário Camboriú & PAS & $\begin{array}{c}27^{\circ} 50^{\prime} \mathrm{S} \text { e } 48^{\circ} 37^{\prime} \mathrm{W} ; 26^{\circ} 59^{\prime} \mathrm{S} \text { e } \\
48^{\circ} 39^{\prime} \mathrm{W}\end{array}$ & $04 / 10 / 2007$ \\
\hline \multicolumn{4}{|l|}{ Guaramirim } \\
\hline SC-413 & PAS & $26^{\circ} 28^{\prime} \mathrm{S}$ e $48^{\circ} 36^{\prime} \mathrm{W}$ & $06 / 10 / 2007$ \\
\hline
\end{tabular}


Polioptila lactea (Sharpe, 1885) (balança-raboleitoso): Esta é uma espécie endêmica da Floresta Atlântica e quase-ameaçada, com registros desde o Espírito Santo até o Rio Grande do Sul, leste do Paraguai e nordeste da Argentina (BirdLife International, 2007). Apresenta registros recentes apenas nas Florestas Estacionais (Decidual e Semidecidual) e na Floresta Ombrófila Mista do interior do continente, no limite sudoeste de sua distribuição (Belton, 1994; Willis e Bosso, 1997; Straube et al., 2004). De acordo com Rosário (1996), não existem registros fidedignos em Santa Catarina, apesar do estado figurar na área de distribuição da espécie (e.g. Ridgely e Tudor, 1989). Encontramos esta espécie na Floresta Ombrófila Densa Montana do
Parque Nacional Serra do Itajaí. A ave foi observada entre 07 e 12 de setembro de 2007 na Fazenda Santa Rita,

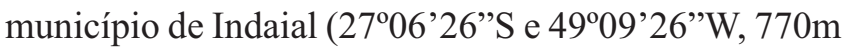
s.n.m.), onde também obtivemos gravações que estão depositadas no arquivo sonoro digital xeno-canto (www. xeno-canto.org), cujo sonograma foi obtido através do programa Syrinx 2.2.1 (Burt, 2006) (Figura 1). A espécie foi encontrada novamente nos dias 07 e 11 de novembro de 2007 na localidade de Ribeirão Jundiá, município de Apiúna (2707'13"S e 49 15 '17'W, 700 m.s.n.m.). Belton (1994) e Willis e Bosso (1997) comentam que a espécie participa de bandos mistos, o que não ocorreu em nossos registros. As aves foram vistas sempre em pares, com o macho vocalizando insistentemente e respondendo prontamente ao playback.

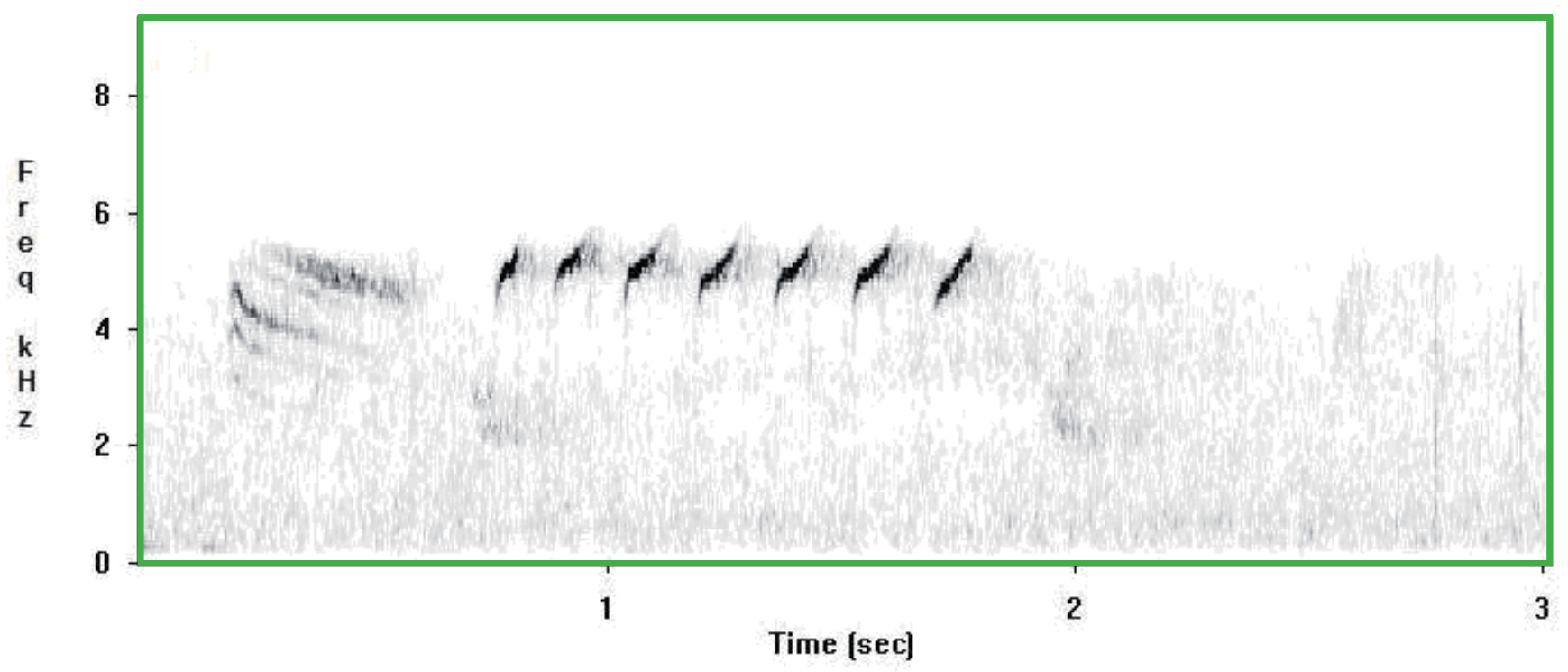

FIGURA 1: Sonograma da vocalização de Polioptila lactea gravada no Parque Nacional da Serra do Itajaí, Indaial, Santa Catarina. Vocalização em resposta ao playback. 


\section{Agradecimentos}

Agradecemos à FATMA e Mobasa S.A. pelo auxílio durante as pesquisas na REBIO Estadual do Sassafrás, e aos programas Pibic/FURB e Pipe/art. 170 pelas bolsas de pesquisa. Ao IBAMA, MMA e ACAPRENA pelo apoio logístico e financeiro aos trabalhos realizados no PARNA Serra do Itajaí. Também a Fernando Rodrigo Tortato, Cláudia Sabrine Brandt e Jaqueline Tonetto por acompanharem algumas saídas de campo.

\section{Referências}

Accordi, I. A.; Vinciprova, G.; Sá, J. C. M.; Witt, A. A.; BarcellosSilveira, A. 2002. Registros notáveis da avifauna de Santa Catarina, Brasil. In: Sales Jr. (org.). Biodiversidade do semi-árido e a conservação das suas aves (incluindo resumos do $\mathrm{X}$ Congresso Brasileiro de Ornitologia), Fortaleza, Brasil, p.102-103.

Amorim, J. F.; Piacentini, V. Q. 2006. Novos registros de aves raras em Santa Catarina, Sul do Brasil, incluindo os primeiros registros documentados de algumas espécies para o Estado. Revista Brasileira de Ornitologia, 14 (2): 145-149.

Amorim, J. F.; Piacentini, V. Q. 2007. Novas áreas de ocorrência de três Passeriformes no sul do Brasil. Lundiana, 8 (1): 69-73.

Azevedo, M. A. G.; Ghizoni-Jr., I. R. 2005. Novos registros de aves para o Estado de Santa Catarina, sul do Brasil. Atualidades Ornitológicas, 126: 9-12.

Bege, L. A. R.; Marterer, B. T. 1991. Conservação da avifauna na região sul do Estado de Santa Catarina, Brasil. FATMA, Florianópolis, Brasil, 54pp.

Belton, W. 1994. Aves do Rio Grande do Sul: distribuição e biologia. Editora Unisinos, São Leopoldo, Brasil, 584pp.

Bencke, G. A. 2001. Lista de referência das aves do Rio Grande do Sul. Fundação Zoobotânica do Rio grande do Sul, Porto Alegre, Brasil, 104pp.

BirdLife International. 2007. Species factsheet: Polioptila lactea. Disponível em $<$ http://www.birdlife.org $>$. Acesso em 2 de outubro de 2007.

Bornschein, M.; Reinert, B. L.; Pichorim, M. 1997. Nota sobre algumas aves novas ou pouco conhecidas no sul do Brasil. Ararajuba, 5 (1): 53-59.

Burt, J. 2006. Programa Syrinx versão 2.6f. Disponível em <http:/ www.syrinxpc.com>. Acesso em 14 de agosto de 2007.

CBRO. 2001. Resolução no 50 - Incluir Picumnus temminckii Lafresnaye, 1845 na Lista Principal de Aves Brasileiras. Nattereria, 2: $55-56$.

CBRO. 2002. Resolução No 86 - Redefinir a abrangência geográfica de Picumnus cirratus Temminck, 1825, da Lista Principal de Aves Brasileiras, por decorrência da Resolução No 50 . Nattereria, 4:1.

De La Peña, M. R.; Rumboll, M. 1998. Birds of southern South America and Antarctica. Princeton University Press, New Jersey, USA, 304pp.
Develey, P. F.; Endrigo, E. 2004. As aves da grande São Paulo: guia de campo. Aves e Fotos Editora, São Paulo, Brasil, 300pp.

Efe, M. A.; Azevedo, M. A. G.; Filippini, A. 2007. Avifauna da Estação Ecológica de Carijós, Florianópolis, SC. Ornithologia, 2(1): $1-13$.

Ferguson-Lees, J; Christie, D. A. 2001. Raptors of the World. Houghton Mifflin Company, New York, USA, 992pp.

Ghizoni-Jr., I. R. 2004. Registro de Polioptila dumicola (Aves: Muscicapidae, Sylviinae) no Estado de Santa Catarina, sul do Brasil. Biotemas, 17 (2): 205-208.

Ghizoni-Jr., I. R.; Silva, E. S. 2006. Registro do saí-canário Thlypopsis sordida (d'Orbigny \& Lafresnaye, 1837) (Aves, Thraupidae) no Estado de Santa Catarina, sul do Brasil. Biotemas, 19 (2): 8182.

Naka, L. N.; Mazar Barnet, J.; Kirwan, G. M.; Tobias, J. A.; Azevedo, M. A. G. 2000. New and noteworthy birds records from Santa Catarina State, Brazil. Bulletin of the British Ornithologists' Club, 120: 237-250.

Naka, L. N.; Mazar Barnet, J.; Kirwan, G. M.; Tobias, J. A.; Buzzetti, D. 2001. Records of bird species previously considered uncommon in Santa Catarina State, Brazil. Cotinga, 16: 68-70.

Naka, L. N.; Rodrigues, M.; Roos, A. L.; Azevedo, M. A. G. 2002. Bird conservation on Santa Catarina Island, Southern Brazil. Bird Conservation International, 12: 123-150.

Piacentini, V. Q.; Campbell-Thompson, E. R. 2006. Lista comentada da avifauna da microbacia hidrográfica da Lagoa de Ibiraquera, Imbituba, SC. Biotemas, 19 (2): 55-65.

Piacentini, V. Q.; Ghizoni-Jr, I. R.; Azevedo, M. A. G.; Kirwan. G. 2006. Sobre a distribuição de aves em Santa Catarina, Brasil, parte I: registros relevantes para o Estado ou inéditos para a Ilha de Santa Catarina. Cotinga, 26: 25-31.

Reichholf, J. 1974. Artenreichtum, Häufigkeit und Diversität der Greifvögel in einigen Gebieten von Südamerika. Journal für Ornithologie, 115 (4): 381-397.

Ridgely, R. S; Tudor, G. 1989. The birds of South America. Volume 1, The Oscine passerines. University of Texas Press, Austin, USA, 516pp.

Rosário, L. A. do. 1996. As aves em Santa Catarina: Distribuição geográfica e meio ambiente. FATMA, Florianópolis, Brasil, 326pp.

Rupp, A. E.; Brandt, C. S.; Fink, D.; Thom-e-Silva, G.; Laps, R. R.; Zimmermann, C. E. 2007. Registros de Caprimulgiformes e a primeira ocorrência de Caprimulgus sericocaudatus (bacurau-rabode-seda) no Estado de Santa Catarina, Brasil. Revista Brasileira de Ornitologia, 15 (4): no prelo.

Sick, H. 1993. Birds in Brazil: a natural history. Princeton University Press, Princeton, UK, 570pp.

Sick, H. 1997. Ornitologia Brasileira. $2^{\mathrm{a}}$ ed. Editora Nova Fronteira, Rio de Janeiro, Brasil, 862pp.

Sick, H.; Rosário, L. A. do.; Azevedo, T. R. 1981. Aves do Estado de Santa Catarina. Lista sistemática baseada em bibliografia, material de museu e observação de campo. Sellowia-Série Zoologia, 1: $1-51$.

Straube, F. C.; Urben-Filho, A.; Kajiwara, D. 2004. Aves. In: Mikich, S. B. \& Bérnils, R. S. (orgs.). Livro vermelho da fauna ame- 
açada de extinção no Estado do Paraná. Instituto Ambiental do Paraná, Curitiba, Brasil, p.143-496.

Straube, F. C.; Urben-Filho, A.; Gatto, C. 2005. A avifauna do Parque Estadual do Cerrado (Jaguariaíva, Paraná) e a conservação do Cerrado em seu limite meridional de ocorrência. Atualidades Ornitológicas, 127: 29.

Willis, E. O.; Bosso, A. 1997. A nest of the Cream-bellied Gnatcatcher, Polioptila lactea. Ornitologia Neotropical, 8: 75-76. 